\title{
Radiological aspects in patients diagnosed with SARS-CoV-2 infection - a literature review
}

\author{
Maria Cristina Hoara ${ }^{1}$, Andreea Florentina Stoenescu ${ }^{1,2}$, Nedeea Duta ${ }^{1}$, \\ Petre Calistru ${ }^{1,2}$, Emanoil Ceausu ${ }^{1,2,3}$, Simin-Aysel Florescu ${ }^{1,2}$ \\ 1"Dr. Victor Babes" Infectious and Tropical Diseases Clinical Hospital, Bucharest, Romania \\ 2"Carol Davila" University of Medicine and Pharmacy, Bucharest, Romania \\ ${ }^{3}$ Academy of Medical Sciences, Romania
}

\begin{abstract}
Currently, SARS-CoV-2 infection has affected the entire population of the globe, registering an alarming increase in the number of cases, but also in deaths.

The main clinical expression of COVID-19 is pneumonia, for its evaluation being necessary an imaging characterization of lung lesions both by chest radiography and by computed tomography (CT) of the chest. Various studies performed on groups of patients revealed that CT examination is more accurately in diagnosing lung lesions at the onset of symptoms, but also before it. The most common lesions were multiple, bilateral, peripheral, in the lower lung fields. The appearance often varies between alveolar consolidation and groundglass. Also, the data from the relevant studies emphasize the possibility of the existence of early pathological aspects on CT acquisitions, which do not involve clinical expression. Thus, it can be stated that both investigations are crucial in assessing the impact of the new coronavirus infection on the lung parenchyma, but also in guiding therapeutic management.
\end{abstract}

Keywords: SARS-CoV-2 infection, radiological aspects, computed tomography (CT)

\section{INTRODUCTION}

Coronaviruses are spherical or pleomorphic, enveloped viruses with a positive RNA genome that belong to the Coronaviridae family. The name comes from the Latin language, the word "corona" means "crown", referring to the appearance of the virus viewed through the electron microscope, at the level of the tire there being glycoprotein projections.

The subfamily Orthocoronaviridae is divided into 4 genera: alpha, beta, gamma, respectively delta coronavirus. Of these, the alpha and beta genera affect mammals, while gamma and delta-coronaviruses mainly affect birds.

In 1965, coronavirus was identified as a pathogen for humans. There are 7 subtypes pathogenic to hu- mans. Their main targets are epithelial cells in the respiratory tract and digestive tract.

Transmission can be both aerogenic through respiratory secretions and fecal-oral. Incubation varies depending on the viral strain, being short in the case of coronaviruses that produce the common cold (on average, 3 days), but going up to 14 days in the case of SARS. Symptomatology can range from asymptomatic to fatal; thus, fever, cough, dyspnea, physical asthenia, but also gastrointestinal manifestations such as diarrhea and vomiting may be present. Rarely, rhinorrhea and odynodysphagia may be present. In severe forms, the lower respiratory tract can also be affected, causing pneumonia and acute respiratory distress syndrome. 
Coronaviruses have a high mutagenic capacity and are distinguished by overcoming the species barrier, including from animals to humans, with the subsequent possibility of interhuman transmission. Thus, in the last 20 years, three such events have taken place, the last one leading to the first coronaviral pandemic, which is currently on the rise. The first epidemic occurred in 2003, caused by SARS-CoV (Severe Acute Respiratoy Syndrome - Coronavirus), later erupting MERS (Middle East Respiratoy Syndrome) in 2012, while today we face SARS-CoV-2, responsible for COVID-19 infection (Coronavirus Disease of 2019).

In December last year, a number of cases of pneumonia of unknown etiology were reported in people in the Chinese region of Hubei. Subsequently, on January 7,2020 , the etiological agent was identified by sequencing the viral genome: 2019 - nCoV (the new coronavirus - strain that had not been previously identified in humans), and on January 11 the first death was recorded. The number of cases of the disease continued to grow alarmingly, gradually affecting other continents (on January 20 the first case was confirmed in the USA, and on January 24 in France). On 12.02.2020, the new coronavirus was named SARSCoV-2, and the associated disease - COVID-19.

\section{IMAGING OF LUNG INJURIES IN COVID-19}

Knowing the substrate of both attachment and intracellular penetration of SARS-CoV-2 virus, namely the ACE2 receptor (angiotensin-converting enzyme 2 ), which is richly represented on the surface of pneumocytes, it can be stated that the main clinical expression of COVID-19 is pneumonia. As a result, imaging characterization of lung lesions is of interest in assessing the impact of the disease at this level, which is not always correlated with symptoms' severity. Moreover, follow-up of the radiological aspect (either with chest radiography or computed tomography) may have a prognostic role. Last but not least, the diagnostic role of CT is debated, as it can sometimes reveal specific tissue damage prior to confirmation of infection through the classical method - detection of viral RNA by RT-PCR.

The first relevant studies on these topics were published in Asia, especially in China, the country that reported the first human cases of this new coronaviral infection. In the following, we will review some of these articles, in order to describe the lung lesions' pattern and its evolution, as well as perform a comparative analysis of images obtained by radiography and chest computed tomography, respectively.

In contrast to CT scan, fewer data concerning classical radiology have been published, given the lower sensitivity of early detection of lung damage due to COVID-19 using the latter, but also because higher resolution imaging has become widely accessible, making detailed and discriminatory lesion description possible within short time. In a study published in late February in South Korea on a group of 9 patients with molecular confirmed disease, all underwent a chest X-ray at the time of hospitalization, but pathological changes were identified in only three cases (33\%). In contrast, CT initially revealed changes in 8 of the 9 cases (approximately 89\%), also demonstrating lesions in the ninth patient at follow-up, one week later (1). Another study conducted in China on a group of 21 symptomatic patients (with one exception) diagnosed with COVID-19 reported a higher success rate in the initial detection of lesions by using chest radiography ( 3 out of 5 cases tested $-60 \%$ ), but this may be due to the small subset of patients who underwent this type of investigation. In comparison, CT-suggestive images were obtained in 19 of the 21 subjects (90\%), including the two patients with non-informative radiographs (2). Although these results are inferred from small groups' analysis, it is well known that $\mathrm{CT}$ scan carries a higher resolution and sensitivity, which also seems to be the case for SARS-CoV-2 pneumonia's early imaging documentation, according to the papers cited above.

Regarding the type of impairments identified by standard radiology, there are several parameters against which they are described: which lung is involved (left/right/bilateral involvement), cephalocaudal distribution (apical/middle/basal region), midlateral distribution (central/peripheral/both), the shape of the lesions (unique, poorly delimited - "flu"/nodular) and their density ("ground-glass"/alveolar consolidation).

The first study shows that two of the three patients had bilateral involvement, exhibiting irregular multilocular opacities, while the third one developed a single nodular lesion within the left lower lung field. In total, 10 distinct pathological images were revealed by chest radiography, being mostly represented by consolidation areas $(70 \%)$, located mainly in the lower lung fields (50\%), peripherally $(80 \%)$. Moreover, 
the three subjects were assessed by daily lung radiography and initial progression to confluent lesions over a 3-4 days period was documented for two of them, followed by improvement over the next days. In the case of the third patient, no significant changes were reported during the 8 days of collecting data (1). Similarly, the second study cited shows that bilateral damage was found in the same proportion (two out of three individuals), in the form of lobar consolidation, mainly located within lungs' bases, but without a predilection for peripheral regions, although the CTs of these same patients subsequently proved a predominantly eccentric disposition (2).

CT imaging often reveals details that cannot be observed with classical radiology and describes the pathological processes more accurately, which is also the case for COVID-19 pneumonia. Unlike the only 10 individual images spotted by radiography, 77 lesions were detected by computed tomography within the South Korean study's group. With respect to their total number, the majority (40, respectively $52 \%$ ) were unique but had the tendency to confluence, whilst the others were of nodular shape. Within the first category, the most common aspect found was a mixture of "ground-glass" opacities and alveolar consolidation (50\%), followed by "ground-glass" as single pattern (35\%). The localization was preferentially peripheral $(75 \%)$, more precisely subpleural, posterior $(70 \%)$ and basal $(60 \%)$. The nodules were mainly of "ground-glass" appearance (57\%) or partially solid
(43\%), with peripheral (81\%) and posterior (65\%) distribution, respectively. Also, most of them were depicted along the bronchovascular bundle (59\%). Referring to patients, 8 of them suffered bilateral involvement, while the most frequently affected lobe was the right lower one (in 8 patients), followed by the left upper and the left lower ones, in that order (1).

Although a description of the entire group of pulmonary pathological changes is useful in understanding the pattern of COVID-19 pneumonia, it does not fully satisfy the need to visualize the individual as a whole, who may associate various lesional types as previously presented. In this light, the work of $\mathrm{Ng}$ et al. also exposes the particular lesion characteristics of the patients submitted to the study group: of the total of 21, two had normal examinations; 11 of those affected showed predominantly "ground-glass" opacities $(>50 \%$ of the abnormal images found in the same patient), 4 of them mainly developed alveolar consolidation ( $>50 \%$ of the abnormal images found in the same patient), whilst the last 4 expressed an equal ratio between the two types of pulmonary opacities. 95\% of the subjects experienced exclusively peripheral involvement. Regarding the vertical axis, the distribution was as follows: 8 patients had lower lobes involvement prevalence, another 8 experienced equal involvement between the upper and lower lung halves, respectively, while in the case of the last 3 patients, lesions of the upper lobes were mainly noticed (2).

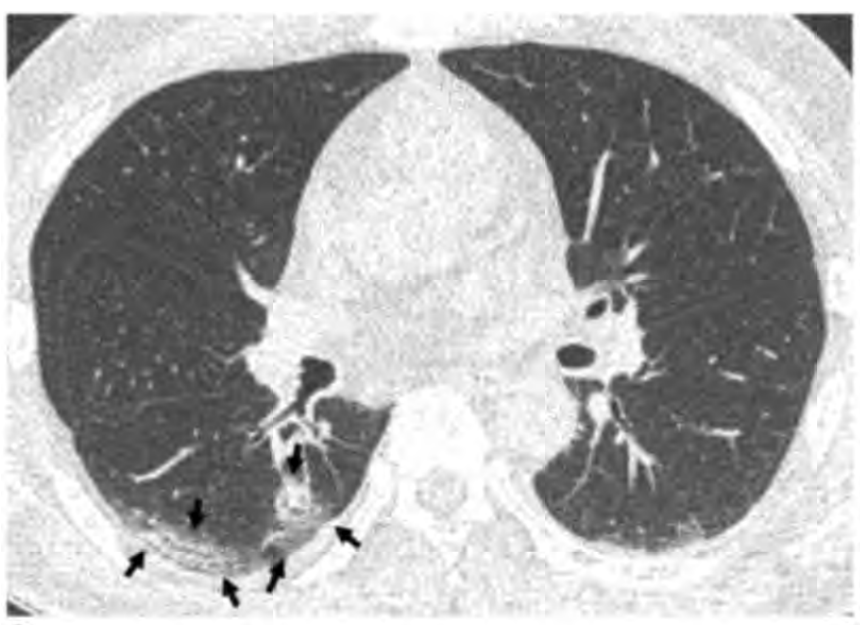

A

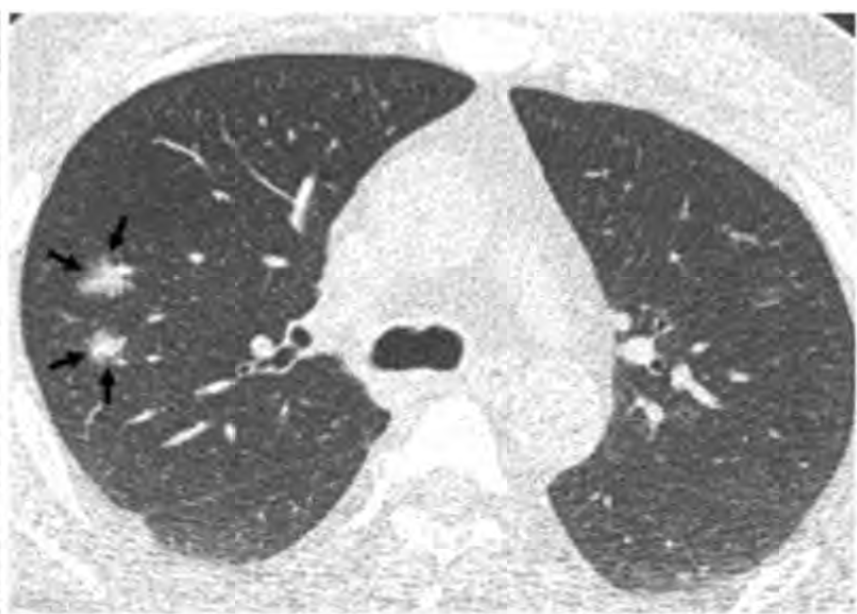

B

FIGURE 1. Representative CT images (A, B) of COVID-19 pneumonia manifesting as radiograph-negative multiple patchy to nodular mixed ground-glass opacities and consolidations.

A. Axial chest CT image shows ill-defined mixed ground-glass opacities and consolidative lesions with patchy and elongated shape (arrows) touching pleura in superior segment of right lower lobe. B. Axial chest CT image shows ill-defined part-solid nodules (arrows; mixed ground-glass opacities and solid nodules) along bronchovascular bundles in posterior segment of right upper lobe (15). 
Beyond the actual description of the radiological picture exhibited by the new coronaviral pneumonia, the inclusion of CT acquisitions in the corroboration between clinical and paraclinical information contributes to assessing disease severity and allows for monitoring the evolution under treatment, as well as even establishing a prognostic link between the dynamics of lung lesions and the outcome of patients.

In this light, the work of Shi et al. (3) makes important clarifications. The study group comprises 81 patients with confirmed infection, having a mean age of 49.5 years, most of whom are male (52\%). It is divided into 4 subgroups depending on the time of the first CT scan performed: in group I individuals whom had been tested prior to the onset of symptoms were included, group II consisted of those tested one week after onset at most, group III included patients tested beyond the first week of illness, but no later than two weeks after onset, whereas the last group enrolled those who had benefited from the investigation between the second and third week of illness. To start with, the study enhances the detection of frequent subclinical condition (15 patients included in the first group, all of them experiencing lesions), a topic discussed here below. Further on, although the lesion characteristics are not entirely comparable (coming from different individuals), the authors consider that this particular division is representative for a model of lung damage evolution during COVID-19 pneumonia. Thus, even if its generic pattern showed mostly bilateral (79\% of patients) and peripheral (54\%) disposition of opacities which were predominantly of "ground-glass" appearance $(65 \%)$, poorly delimited (81\%) and especially involved the right lower lobe, a picture largely similar to the one described in the studies mentioned above, these aspects differ along the clinical course of the disease. If within the subgroup of asymptomatic subjects the lesions were predominantly unilateral (60\%) and multifocal (53\%), represented mainly by "ground-glass" opacities (93\%), they subsequently became more diffuse, frequently bilateral, being gradually replaced by pulmonary consolidation and mixed aspects. In addition, interstitial fibrosis was observed towards the end of illness, as well as pleural thickening or pleural effusion, the latter being cited in the work of Lin et al., as well (4).

The authors also performed a proper imaging follow-up, as 57 patients underwent serial CT investigations and the results were grouped into 4 possible ways of evolution: 1 - initial progression, followed by improvement ( $46 \%$ of them, of whom $92 \%$ were eventually discharged), 2 - deterioration (32\%, with two patients later succumbing to the disease $-11 \%$ of those belonging to this category), 3 -improvement ( $14 \%$ of patients, most of whom have been discharged), respectively 4 - steady state $(9 \%$, all remaining hospitalized at the end of data collection). As a result, the authors concluded that, in addition to other variables considered in the study (old age, male gender, associated comorbidities), worsening radiological picture could be a risk factor for poor prognosis in patients with COVID-19 pneumonia. However, the data should be interpreted with caution, taking into account that 9 of the 81 patients $(11 \%)$ suffered from a pre-existing chronic lung pathology, which could have influenced the lesional characteristics observed in this paper and led to bias of results (3).

In an effort to better quantify lesions observed with the aid of CT, Pan et al. (5) propose a semiquantitative score of lung damage, by approximating its degree of extension. Thus, each of the 5 lobes was rated from 0 to 5 , corresponding to a percentage of lung involvement within the respective lobe, with a maximum possible total CT score of 25 . In plus, the authors monitored the variation in time of the lesional type. Thus, despite exclusion of patients with severe disease forms was acknowledged as a study limit, they came up with the result that the majority of subjects included in the group (18 of a total of 21, representing $85 \%$ ) reached the highest degree of lung involvement (quantified as 7 points in average, but up to 14 in one patient) at around 10 days after the onset of symptoms. With regard to the lesions' characteristics, subpleural "ground-glass" was initially mostly observed, which progressed in size along with the appearance of crazy-paving aspect ("ground-glass" combined with both intra- and interlobular septal thickening images), following that on days 9 to 13 from the beginning of illness, the lesions be predominantly replaced by alveolar consolidation. From the 14 th day on, the gradual resorption of the infiltrates was noticed, but not without the remanence of a discreet residual "ground glass" appearance. The authors noted incomplete resolution of pathological images at the end of data collection (26 days from onset), which may not necessarily argue for persistent lesions over time, but rather for the practice of longer follow-up leading to objective results on the duration of pulmo- 
nary involvement in SARS-CoV-2 infection. Related to this topic, regression and disappearance of lesions on CT examination were reported in 24 (42\%) of 57 patients even before they became negative on RTPCR analysis for viral RNA detection, in a study conducted by Ai et al. (6).

In Europe, one of the most affected countries is Italy, with about 200,000 cases and over 25,000 deaths at the time of writing this paper. Caruso et al. conducted a prospective study to monitor the accuracy of the CT scan. Of the 158 patients admitted to the hospital over two weeks, 62 were diagnosed with COVID-19 infection. Of these, 60 had pulmonary changes on CT examination (the other two were excluded due to the presence of movement artifacts). The results confirmed the conclusions of Asian studies related to the predilection of the type of damage and the location of the lesions. Thus, opacity in matte glass was identified among all patients, posterior multilobular involvement was present in $93 \%$ of cases, and bilateral localization was highlighted in $91 \%$ of cases. Simultaneous involvement of all lung lobes was present in 43 of the patients (74\%). No significant differences were identified at the $\mathrm{CT}$ examination between hospitalized patients with severe disease and those with mild form, isolated at home (7).

Returning to the topic of identifying imaging changes amongst asymptomatic individuals molecularly diagnosed with COVID-19, many papers discuss the matter, objectifying early pathological aspects on $\mathrm{CT}$ acquisitions which do not bear a clinical expression. In addition to the retrospective study discussed above (3), similar observations were made in the case study of Lin et al. (4), particular in that it documented pleural effusion for the first time in connection with the new coronaviral pneumonia. Yet another mention appears in the paper of $\mathrm{Hu}$ et al. (8) on 24 asymptomatic close contacts later confirmed positive, of whom $50 \%$ showed typical "ground-glass" changes.

Given these reports, it appears that informative CT examination may precede the onset of symptoms, which raises the question upon routine use of CT as for early description of pulmonary tissue impact laid by the new coronavirus infection, with the potential of guiding early therapeutic gestures in an effort to improve patient outcome.

However, a large study from China (9) noted the possibility of a lack of informative imaging data (both by standard radiology and CT) in 157 of 877 (approx- imately $18 \%$ ) individuals with non-severe disease, but also in 5 of 173 patients (approximately 3\%) with severe form of the disease, which questions both the substrate that led to clinical expression (especially regarding the severe form), but also the sensitivity of the test.

Last but not least, there is controversy upon the fact that computed tomography could replace molecular testing in pursuit of diagnosing COVID-19. Although the globally accepted case definition stated by the World Health Organization provides confirmation by viral RNA detection through RT-PCR only, a study of 1,014 patients in Wuhan (6) claims that CT sensitivity is higher than that of PCR in early detecting those infected, also showing that the duration of obtaining a result is much shorter. However, further studies are needed to determine exactly whether an imaging criterion could confirm SARS-CoV-2 infection. We must also not forget that the $\mathrm{CT}$ technique is not so widespread within developing countries.

More recent scientific opinions oppose the tendency of using CT as a screening tool for COVID-19 pneumonia basing on valid arguments, omitted by studies that encourage this practice. Emphasizing the importance of applying a more elaborate working methodology and subjecting research to a thorough and objective peer-review, Hope and colleagues (10) believe that in the current pandemic context the need of medical community for solutions and answers to questions about the diagnosis and treatment of COVID-19 leads to publication of premature results, not rigorously verified, with potentially unfavorable consequences in clinical practice. They bring to attention several issues, including the lack of lesions' specificity described by CT in this disease (the appearance of "ground-glass" and alveolar consolidation), which may be the imprint of many other preexisting diseases, both infectious and non-infectious. Therefore, a correct approach would be to associate the lesions with the new coronaviral infection only amongst those with a high pre-test probability of suffering from this condition. In addition, the widespread use of CT should involve strict measures to prevent transmission of SARS-CoV-2 among both patients and healthcare professionals working in the imaging department. Last but not least, another comment is made upon a study that stated both increased sensitivity and specificity in differentiating lung lesions of COVID-19 from those given by other viral infections, 
identifying errors in design execution which could have leaded radiologists who interpreted the images towards a specific diagnosis. In addition, the degree of subjectivism of observers in assessing lesions is emphasized. In support of these ideas, Huang et al. (11) also published a similar scientific opinion in The Lancet, further noting the risk of irradiation without a definite benefit, asking for establishment of clear indications for the use of this investigation.

A new alarming observation has the potential of changing attitudes regarding imaging in SARS-CoV-2 infection, namely the recognition of pulmonary embolism as a result of viral pathogenic mechanisms. Grillet et al. (12) identified 23 cases of acute pulmonary embolism among 100 patients (representing $23 \%$ ) with severe forms of disease undergoing contrast CT scan. This diagnosis was more frequently associated with admission to the Intensive Care Unit and establishment of mechanical ventilation when compared to the subgroup of 77 patients without a thrombotic event. These observations require a review of the therapeutic regimens applied, in order to include anticoagulant medication under proper indication.

Although the focus of the pandemic created by SARS-CoV-2 is directed at adults, children are also affected to a lesser extent, respecting the data validated so far. Thus, a study performed on a group of 15 children diagnosed with COVID-19 analyzed the distribution and morphology of lung lesions revealed by CT examination. Of these, 6 patients did not show any change at the first examination, and 9 of the patients had inflammatory lesions. Of these, 7 showed opacity in nodular ground glass, and in the case of the other 2 , the opacity had an irregular surface. Although they followed the appropriate treatment, 6 patients being declared negative, at another CT examination 2 of them showed a reduction of lesions, and in the case of one patient no improvement was observed. Out of the 9 patients who were still positive, new small lesions in ground glass were found in 3 of them (13). Moreover, another retrospective study performed on a group of 171 children confirmed for infection with the new coronavirus noticed CT lung damage among 12 of the asymptomatic ones. In addition, the main lesion type identified was, similar to adults, represented by opacity in ground glass (14).

\section{CONCLUSIONS}

Both lung radiography and CT examination are undoubtedly crucial investigations in assessing the impact of the new coronavirus infection on lung parenchyma, but also in guiding therapeutic management, with further studies and expert consensus working on establishing clear indications of their use in order to maximize the benefit in detriment of risks for the patient. Moreover, we consider that studies are needed regarding the characteristics of lung damage produced by SARS-CoV-2 in relation to other viral etiological agents, as long as distinctive elements will come to support the diagnosis of this particular pneumonia.

Conflict of interest: none declared Financial support: none declared

\section{REFERENCES}

1. Yoon SH, Lee KH, Kim JY, Lee YK et al. Chest Radiographic and CT Findings of the 2019 Novel Coronavirus Disease (COVID-19): Analysis of Nine Patients Treated in Korea. Korean Journal of Radiology. 2020;21(4):494-500.

2. Ng MY, Lee EY, Yang F, Li X, Wang H, Lui MM, Lo CSY et al. Imaging Profile of the COVID-19 Infection: Radiologic Findings and Literature Review. Radiology: Cardiothoracic Imaging. 2020;2(1).

3. Shi H, Han X, Jiang N, Cao Y, Alwalid O, Gu J, Fan Y, Zheng C. Radiological findings from 81 patients with COVID-19 pneumonia in Wuhan, China: A descriptive study. The Lancet Infectious Diseases. 2020;20(4):425-434

4. Lin C, Ding Y, Sun Z, Li X, Chen Z, Niu M. Asymptomatic novel coronavirus pneumonia patient outside Wuhan: The value of CT images in the course of the disease. Clinical Imaging. 2020;63:7-9.

5. Pan F, Ye T, Sun P, Gui S, Liang B, Li L et al. Time Course of Lung Changes On Chest CT During Recovery From 2019 Novel Coronavirus (COVID-19) Pneumonia. Radiology. 2020.

6. Ai T, Yang Z, Hou H, Zhan C, Chen C, Lv W, Tao Q, Sun Z, Xia L. Correlation of chest CT and RT-PCR testing in coronavirus disease 2019 (COVID-19) in China: A report of 1014 cases. Radiology. 2020.
7. Caruso D, Zerunian M, Polici M, Pucciarelli F., Polidori T., Rucci C et al. Chest CT Features of COVID-19 in Rome, Italy. Radiology. 2020.

8. Hu Z, Song C, Xu C, Jin G, Chen Y, Xu X, Ma H et al. Clinical characteristics of 24 asymptomatic infections with COVID-19 screened among close contacts in Nanjing, China. Science China Life Sciences. 2020.

9. Guan W, Ni Z, Hu Y, Liang W, Ou C, He J et al. Clinical Characteristics of Coronavirus Disease 2019 in China. The New England Journal of Medicine. 2020.

10. Hope MD, Raptis CA, Henry TS. Chest Computed Tomography for Detection of Coronavirus Disease 2019 (COVID-19): Don't Rush the Science. Annals of Internal Medicine. 2020.

11. Huang Y, Cheng W, Zhao N, Qu H, Tian J. CT screening for early diagnosis of SARS-CoV-2 infection. The Lancet Infectious Diseases. 2020.

12. Grillet F, Behr J, Calame P, Aubry S, Delabrousse E. Acute Pulmonary Embolism Associated with COVID-19 Pneumonia Detected by Pulmonary CT Angiography [published online ahead of print, 2020 Apr 23]. Radiology. 2020;201544. 
13. Feng K, Yun YX, Wang XF, Yang GD, Zheng YJ, Lin CM, Wang LF. Analysis of CT features of 15 children with 2019 novel coronavirus infection Zhonghua Er Ke Za Zhi. 2020;58(0):E007.

14. Lu X, Zhang L, Du H, Zhang J, Li YY, Qu J, Zhang W, Wang Y, Bao S, Li Y, Wu C, Liu H, Liu D, Shao J, Peng X, Yang Y, Liu Z, Xiang Y,
Zhang F, Silva RM, Pinkerton KE, Shen K, Xiao H, Xu S, Wong GWK; Chinese Pediatric Novel Coronavirus Study Team. SARSCoV-2 Infection in Children. N Engl J Med. 2020;382(17):1663-1665. 15. https://www.ncbi.nlm.nih.gov/pmc/articles/PMC7082662/figure/F4/. 